\title{
Der Informationsbedarf von Patienten mit Krebserkrankungen in Deutschland - eine Befragung von Patienten und Angehörigen
}

\author{
I. Rudolph ${ }^{1}$, E. Seilacher ${ }^{1}$, M.-J. Köster ${ }^{1}$, J. Stellamanns ${ }^{1}$, P. Liebl ${ }^{1}$, J. Zell², S. Ludwig ${ }^{3}$, V. Beck ${ }^{4}$, J. Hübner ${ }^{5}$
}

Einleitung | Patienten mit Krebserkrankungen haben einen hohen krankheitsspezifischen Informationsbedarf. Tumorpatienten bevorzugen den behandelnden Arzt als Quelle, wenden sich mit ihren Fragen aber auch an Laien oder Informationsmedien. Ziel der vorliegenden Untersuchung war es, aktuelle Daten für Deutschland zu Informationsbedürfnissen und Informationszufriedenheit aus Patienten- und Angehörigensicht zu gewinnen.

Methodik und Teilnehmer | Mittels eines standardisierten Fragebogens wurden 226 Patienten und 32 Angehörige, die an Patientenveranstaltungen teilnahmen, befragt.

Ergebnisse I Die Zufriedenheit mit bisher erhaltenen Informationen ist generell hoch. Das direkte Gespräch mit dem Arzt ist die bedeutsamste Informationsquelle, insbesondere für Patienten über 60 Jahre. Am zweitwichtigsten sind Selbst- hilfegruppen gefolgt von interaktiven Medien wie Internet und Online-Foren, die jedoch bei der Gruppe der über 60-Jährigen wenig Bedeutung haben. Als wichtige Eigenschaften einer Informationsquelle werden individuelle und umfassende Informationen durch Experten, die Möglichkeit, Fragen zu stellen und ein konstanter Ansprechpartner genannt. Empathie bzw. Anteilnahme werden dagegen selten als wichtig eingeschätzt. Geschlecht und Alter haben auf diese Präferenzen der Eigenschaften einer Informationsquelle keinen Einfluss.

Diskussion I Der Patienten- und Angehörigenwunsch nach einer individuellen, umfassenden Beratung mit hohem Expertenwissen aus einer Hand zeigt, dass es angesichts der Grenzen der zur Verfügung stehenden Ressourcen in Zukunft immer wichtiger wird, unterstützende Informationsmaterialien für verschiedene Patientengruppen aus zuverlässigen Quellen zu schaffen.

\section{Einleitung}

Krebs ist eine der häufigsten Erkrankungen in Deutschland. 2012 erkrankten 490000 Menschen neu an einem Malignom [16]. Die Prävalenz bösartiger Neubildungen steigt mit der zunehmenden Inzidenz aufgrund des demographischen Wandels, einer früheren Diagnose durch Screening und Früherkennung sowie verbesserter Therapieoptionen, die nicht nur zu einer Heilung, sondern vielfach auch zu einem langen bis chronischen Krankheitsverlauf führen können $[2,5]$.

Immer speziellere diagnostische Methoden und komplexer werdende Therapieabläufe bedeuten nicht nur für den Arzt eine Herausforderung, sondern fordern auch den Patienten. In einer Situation, die manche als lebensbedrohlich wahrnehmen, wird die Beteiligung an Entscheidungsprozessen (Shared Decision Making) und die Übernahme von Verantwortung in der Therapie erwartet (Ziel 13 im Nationalen Krebsplan; [14]).

Patienten mit Krebserkrankungen haben einen hohen krankheitsspezifischen Informationsbedarf. Auch für Tumorpatienten ist das Internet mittlerweile eine wichtige Informationsquelle, allerdings bevorzugen sie das persönliche Arztgespräch $[6,7,12,15]$. In einigen Studien wurden auch Familie und Freunde sowie Medien an erster Stelle als Informationsquelle genannt $[7,13]$.

Über die Anforderungen an Informationsquellen aus der Sicht von Krebspatienten wurde in Deutschland noch relativ wenig geforscht. Grundsätzlich sind Vertrauenswürdigkeit, leichte Auffindbarkeit und Aktualität wichtig [10]. Die wichtigsten Themen, die im Internet recherchiert werden, sind Krebsnachsorge, Krebsvorsorge und Früherkennung, Behandlungsfolgen, Nebenwirkungen und Begleitsymptome, Überlebenschancen, Risikofaktoren und Ursachen (die Reihenfolge entspricht der Bedeutung, Daten aus einer Untersuchung von 2012 in Deutschland [6]). International werden v.a. die Suche nach Therapieoptionen, Folgen der Erkrankung und Behandlungsmöglichkeiten von Nebenwirkungen angegeben [6]. Für einige Patienten steht auch die Frage nach den Handlungsoptionen bei fehlendem Therapieansprechen im Vordergrund [3].

Die Deutsche Krebshilfe und die Deutsche Krebsgesellschaft bieten Patienten eine Reihe von Informationsmöglichkeiten an. Hierzu gehören eine telefonische Beratung durch die Deutsche Krebshilfe, die persönliche oder telefonische Beratung in psychosozialen Beratungsstellen der Landeskrebsgesellschaften, Broschüren der Deutschen
Institute

1 Deutsche Krebsgesellschaft, Berlin

2 Interaktiv GmbH - Creative Information Solutions, Hürth

3 Deutsche Krebshilfe, Bonn

4 Fachbereich Informatik und Medien

Fachhochschule Brandenburg

5 Dr. Senckenberg Chronomedizinisches Institut, J.W. Goethe Universität Frankfurt

\section{Korrespondenz}

PD Dr. med. Jutta Hübner Arbeitsgruppe Integrative Onkologie Dr. Senckenberg Chronomedizinisches Institut J.W. Goethe Universität Theodor-Stern-Kai 7 60590 Frankfurt Tel. 030/322932951 Fax: 030/322932966 E-Mail: huebner@med. uni-frankfurt.de 


\begin{tabular}{lll} 
Parameter & Auswahl & Anzahl (\%) \\
\hline Betroffenenstatus & Patient während Therapie & $108(41,9 \%)$ \\
& Patient nach Therapie & $118(45,7 \%)$ \\
& Angehöriger & $32(12,4 \%)$ \\
& Fehlende Angaben & keine \\
Alter & $\leq 40$ & $8(3,1 \%)$ \\
& $41-60$ & $74(28,7 \%)$ \\
& $\geq 61$ & $172(66,7 \%)$ \\
Geschlecht & Fehlende Angaben & $1,5 \%$ \\
Bildungsabschluss* & weiblich & $169(65,5 \%)$ \\
& männlich & $83(32,3 \%)$ \\
& Hauptschule & $22(8,5 \%)$ \\
& Realschule & $41(15,9 \%)$ \\
& Gymnasium & $55(21,3 \%)$ \\
& FH/Uni & $31(12,0 \%)$ \\
& Fehlende Angaben & $109(42,2 \%)$ \\
& &
\end{tabular}

Tab. 1 Daten zu Betroffenenstatus, Alter, Geschlecht und Bildungsabschluss.

Abb. 1 Bedeutung der Informationsquellen aus Sicht der Befragten (Prozent der Nennungen, Mehrfachnennungen waren möglich). *Direktes Gespräch mit dem Arzt oder der Pflegekraft.

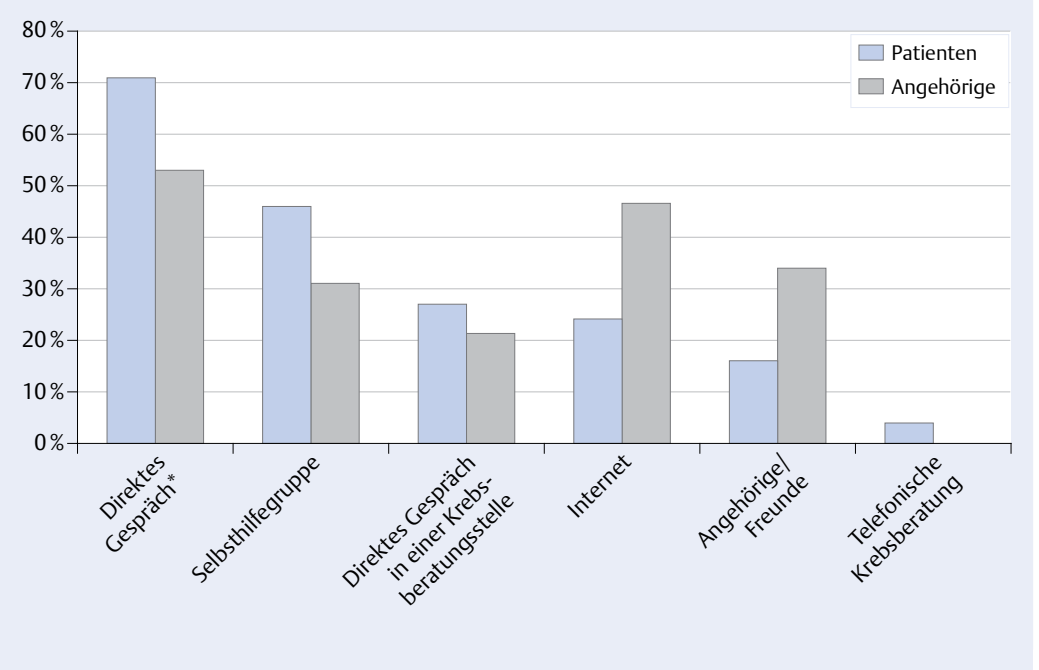

Veranstaltungen wie z.B. Patiententagen und von Informationsmaterialien.

\section{Methodik}

Mittels eines standardisierten Fragebogens wurden anonym die Teilnehmer von folgenden Veranstaltungen befragt:

- zwei große Veranstaltungen der Deutschen Krebshilfe und der Deutschen Krebsgesellschaft

- Offene Krebskonferenz im September 2013 in Dresden: gemeinsame Veranstaltung von Deutscher Krebshilfe, der Sächsischen Landeskrebsgesellschaft und der Deutschen Krebsgesellschaft sowie

- Krebsaktionstag im Februar 2014 in Berlin: gemeinsame Veranstaltung von der Deutschen Krebshilfe, der Berliner Landeskrebsgesellschaft und der Deutschen Krebsgesellschaft

- zwei kleinere Veranstaltungen anderer Anbieter

- Patientenabend der Fachhochschule Brandenburg/Havel im November 2013 und

- Rehabilitationsklinik Bad Salzelmen im November 2013

Der Fragebogen ist von Experten der Deutschen Krebsgesellschaft aufgrund einer systematischen Literaturrecherche zum Thema Informationsbedarf bei onkologischen Patienten entwickelt worden. Die erste Version wurde mit einer kleinen Gruppe von Patientenvertretern diskutiert und nach ihren Rückmeldungen angepasst. Zusätzlich wurde der Fragebogen in einer Selbsthilfegruppe auf Verständlichkeit, Zeitbedarf und Ausfüllungsgrad getestet.

Der Fragebogen umfasste 5 Fragen:

1. Demographische und krankheitsbezogene Daten (Betroffenenstatus, Alter, Geschlecht, Bildungsabschluss, Tumorart)

2. Zufriedenheit mit den bisherigen Informationen (Einschätzung auf einer Ordinalskala entsprechend Schulnoten von 1 bis 6 )

3. Präferierte Informationsquelle (freie Antwort oder Listenauswahl)

4. Wichtigste Eigenschaften des Informationsangebotes (Auswahl von drei Punkten aus einer Liste von vorgegebenen Qualitäten)

5. Vermisste Punkte bei bisher genutzten Informationsquellen (Freitext).

Die Teilnehmer wurden vor Beginn der Veranstaltung und in den Pausen von geschulten Mitarbeitern angesprochen und bei Einverständnis befragt. Die Zeit, die zum Beantworten der Fragen benötigt wurde, lag bei 2 bis 5 Minuten. Die Teilnahme war freiwillig und anonym.

Aufgrund der Anonymität war nach den Vorgaben der Ethikkommission des Universitätsklinikums 
Frankfurt/Main keine Einholung eines Ethikvotums erforderlich.

Die Auswertung der Bögen erfolgte nach Erfassung in IBM SPSS Statistics 20 mittels einer Analyse der Häufigkeiten und der Zusammenhänge (Chi-Quadrat-Test).

\section{Ergebnisse}

Insgesamt nahmen 258 Teilnehmer (226 Patienten und 32 Angehörige) der Veranstaltungen an der Befragung teil; alle Fragebögen waren auswertbar. Die Daten zu Betroffenenstatus, Alter, Geschlecht, Bildungsabschluss und Tumorart sind in $\downarrow$ Tab. 1 zusammengefasst. Die häufigsten Tumorarten waren

- Brustkrebs (90 Patienten; 35,9\%),

- Prostatakrebs (46; 17,8\%),

- Darmkrebs (15; 5,8\%),

- Lungenkrebs $(10 ; 3,9 \%)$ und

- Lymphom oder Leukämie (23 Patienten; 8,9\%). Insgesamt wurden 35 verschiedene Tumorarten primär genannt.

Die Zufriedenheit mit der bisher erhaltenen Information war bei den meisten Teilnehmern hoch

- Note 1: 77 Nennungen $(29,8 \%)$

- Note 2: 127 Nennungen (49,2\%).

Nur 8 Befragte antworteten mit Note 4, 5 oder 6 $(3,1 \%)$. Frauen sind eher unzufrieden mit den erhaltenen Informationen als Männer $(p=0,029)$, Angehörige sind unzufriedener als Patienten $(\mathrm{p}<0,001)$.

Die Bedeutung der verschiedenen Informationsquellen gibt $\rightarrow$ Abb. 1 wieder. Das direkte Gespräch mit dem Arzt ist für Patienten wie Angehörige die bedeutsamste Informationsquelle. An zweiter Stelle stehen bei den Patienten die Selbsthilfegruppen, bei den Angehörigen das Internet (inklusive Online-Foren). Während für die Angehörigen das Gespräch im Familien-und Freundeskreis an dritter Stelle steht, ist es für Patienten das Gespräch in einer Krebsberatungsstelle. Telefonische Beratungen haben in beiden Gruppen keinen hohen Stellenwert. Die Unterschiede bei der Bedeutung des direkten Gespräches mit dem Arzt bzw. mit Famillienangehörigen und Freunden sind signifikant ( $p=0,004$ bzw. $p=0,013)$, die bei der Internetnutzung ist es nicht $(p=0,10)$.

Teilnehmer über 60 Jahre messen dem persönlichen Gespräch eine signifikant höhere Bedeutung zu als die jüngeren Altersgruppen $(p=0,005)$, dagegen hat das Internet eine wesentlich geringere Bedeutung ( $p<0,019) .74,4 \%$ der Teilnehmer aus der Altersgruppe der über 60-Jährigen halten das Internet für unwichtig, nur 25,6\% für wichtig. Geschlecht und Bildungsabschluss haben keinen Einfluss auf die Einschätzung der Bedeutung des Gespräches. Die Nutzung des Internets ist bei Pa-

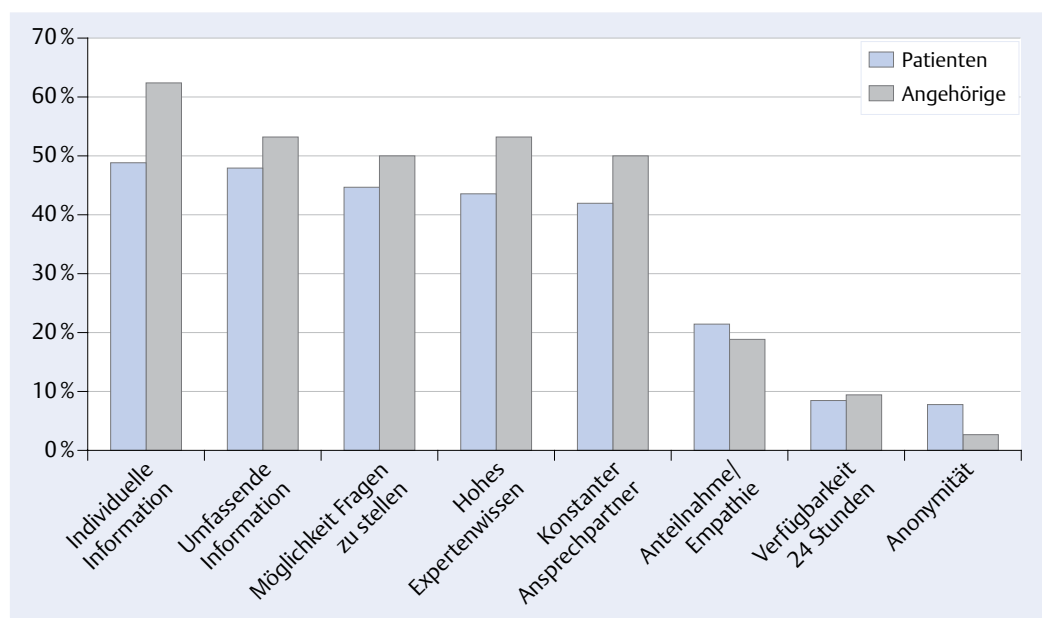

Abb. 2 Eigenschaften von Informationsquellen und ihre Bedeutung für Patienten und Angehörige (Prozent der Nennungen, Mehrfachnennungen waren möglich).

Für Patienten sind die Selbsthilfegruppen bedeutsamer als für Angehörige ( $p=0,013$ ). Frauen geben signifikant häufiger Selbsthilfegruppen als Informationsquelle an als Männer ( $p=0,019)$.

Bei der Auswahl der für eine Informationsquelle wichtigen Eigenschaften stehen individuelle und umfassende Informationen von Experten im Vordergrund. Ebenso sind die Möglichkeit, Fragen zu stellen und ein konstanter Ansprechpartner von Bedeutung. Empathie bzw. Anteilnahme wurde dagegen seltener genannt. Anonymität und 24 Stunden Erreichbarkeit sind von geringer Bedeutung. Die Ergebnisse der Befragung sind im Detail in $>$ Abb. 2 wiedergegeben. Für alle Punkte findet sich kein Unterschied in der Einschätzung durch Patienten und Angehörige. Auch bei diesem Punkt haben Geschlecht und Alter kaum einen Einfluss auf die Prioritäten der Teilnehmer. Umfassende Informationen und hohes Expertenwissen werden von Teilnehmern mit niedrigerem Bildungsabschluss seltener genannt als von Teilnehmern mit hohem Abschluss.

\section{Diskussion}

Unsere Befragung von Teilnehmern von vier verschiedenen Informationsveranstaltungen für Patienten mit Krebserkrankungen ergibt eine hohe Zufriedenheit mit den für die Betroffenen verfügbaren und von diesen genutzten Informationen (-squellen).

Das direkte Gespräch mit dem Arzt oder der Pflegekraft ist die bedeutsamste Informationsquelle, gefolgt von Selbsthilfegruppen und dem Internet bzw. Online-Foren. Dabei ist zu berücksichtigen, dass bei den über 60-Jährigen zwei Drittel das Internet für unwichtig halten. Individuelle und umfassende Informationen aus Expertenhand sind ebenso wichtig, wie die Möglichkeit, Fragen zu stellen und einen konstanten Ansprechpartner 
zu haben. Interessanterweise wurden Empathie bzw. Anteilnahme selten als wichtige Punkte genannt.

Bei der Interpretation und Verallgemeinerung der Ergebnisse sind die Limitationen der Studie zu beachten. Zum einen ist die Teilnehmerzahl (insbesondere bei den Angehörigen) relativ niedrig. Zum anderen handelte es sich bei den Teilnehmern um Patienten und Angehörige, die aktiv an einer Informationsveranstaltung teilnehmen, also ein Kollektiv motivierter und an Informationen interessierter Personen. Dies kann sowohl auf das bisherige Informationsverhalten wie auf die Einstellung zur Art und Weise der Vermittlung der Inhalte aus unterschiedlichen Quellen Einfluss haben. Die Organisation und Bekanntgabe der Veranstaltungen erfolgte in Kooperation mit den örtlichen Selbsthilfegruppen, sodass ein Bias zugunsten der Selbsthilfe zu erwarten ist. Außerdem können Menschen mit körperlichen Einschränkungen nur begrenzt oder gar nicht an solchen Veranstaltungen teilnehmen, sodass die Gruppe der schwerkranken Patienten, die sich im Informationsverhalten unterscheiden könnte, vermutlich unterrepräsentiert ist. Methodisch handelt es sich um keinen validierten, aber um einen eng mit Patientenvertretern abgestimmten Fragebogen. Ursprünglich hätten wir gern weiterführende Fragen in die Befragung mit aufgenommen, um die Themen zu vertiefen. Allerdings stellte sich bereits in der Diskussion mit den Selbsthilfegruppen heraus, dass der gewählte Umfang des Fragebogens als noch akzeptabel beurteilt wurde. Dies bestätigte sich dann bei der Testung an den Patienten (und deren Angehörigen).

Trotz dieser Einschränkungen ist das Ergebnis in Bezug auf die jetzt schon für Patienten und Angehörige zur Verfügung stehende Information positiv. Dabei ergeben unsere Daten auch keine Hinweise auf eine schlechtere Versorgung bestimmter Personengruppen - zwar sind Frauen etwas unzufriedener als Männer, aber dieser Unterschied ist nur grenzwertig signifikant. Die Teilnehmer wiesen unterschiedliche Bildungsabschlüsse auf, dies scheint aber keinen Einfluss auf die Zufriedenheit zu haben. Allerdings ist es denkbar, dass sehr bildungsferne Betroffene Informationsveranstaltungen nicht besuchen und von daher in unserer Studie unterrepräsentiert sind. Gleiches ist bei Personengruppen anzunehmen, die aufgrund von sprachlichen Barrieren von Informationsveranstaltungen in deutscher Sprache nur eingeschränkt profitieren können.

In Bezug auf die Informationsquellen bestätigt unsere Untersuchung die bereits publizierte Vorrangstellung des Arztes bzw. der Pflegekraft. Andere Betroffene, Familie und Medien haben eine wichtige ergänzende Funktion, auch dies entspricht internationalen Daten [7, 13]. Auffal- lend ist die erwähnte hohe Bedeutung der Selbsthilfe, die in anderen Untersuchungen eher eine marginale Rolle spielt, aber mit dem Setting unserer Befragung teilweise erklärt werden kann [12]. Interessant ist die niedrige Bedeutung der Krebsberatungsstellen und der telefonischen Beratungsmöglichkeiten. Ob dies an einem geringen Bekannheitsgrad der Angebote liegt oder damit zusammenhängt, dass diese Angebote nur schwer den gewünschten Anforderungen nachkommen können (eine individuelle ärztliche Beratung ist z.B. nicht möglich), kann aus der Befragung nicht geschlossen werden.

Bemerkenswert ist die geringe Bedeutung von Anteilnahme bzw. Empathie in der Informationsvermittlung. Im Allgemeinen werden diese Merkmale als für die Kommunikation des Arztes mit dem Patienten wesentlich angesehen. Sie führen zu einer höheren Zufriedenheit des Patienten mit dem Gespräch [9]. Für dieses geringe Ranking in der Umfrage kann es verschiedene Gründe geben. Ein Grund dürfte auch hier das Setting sein: Auf einer Informationsveranstaltung könnte der Fokus der Befragten eher auf der reinen Informationsvermittlung liegen. Es ist auch möglich, dass die Patienten durch die vorausgehenden Fragen eher Informationsquellen außerhalb des Gespräches vor Augen hatten, wo Anteilnahme nur indirekt zu vermitteln ist. Eine dritte Erklärungsmöglichkeit könnte sein, dass Empathie in der akuten oder der weit fortgeschrittenen Krankheitssituation bedeutsamer ist als in anderen Situationen. In akuter Therapie befanden sich aber nur etwa ein Drittel der Teilnehmer. Trotzdem stellt sich die Frage, ob sich nicht - auch angestoßen durch die vielfältige Diskussion über Entwicklungen im Gesundheitswesen - die Erwartungshaltung der Patienten verschiebt und der Arzt zunehmend als Experte in sachlich-fachlicher und weniger in zwischenmenschlicher Sicht gesehen wird. In ihrem Review zur Bedeutung der Empathie des Arztes fanden Lelorain et al. [9], dass die Empathie zwar die Belastung der Patienten senkt aber keinen Einfluss auf medizinisches Wissen und Coping hat.

Institutionen, die sich vor Ort, in ihrer eigenen Einrichtung oder in einer Region für Patienteninformation engagieren wollen, können die Ergebnisse unserer Studie nutzen, um ihr Informationsangebot für Patienten zu gestalten. Eine individuelle, umfassende Beratung mit hohem Expertenwissen aus einer Hand kann an die Grenzen der zur Verfügung stehenden Ressourcen stoßen. Deshalb wird es in Zukunft immer wichtiger werden, unterstützende Informationsmaterialien und -quellen anzubieten $[7,15]$. So könnte es auch gelingen, dem relativ breit gefächerten Informationsbedarf ausgehend von der Prävention über Diagnostik und Therapie, supportive und palliative Therapie und begleitende Maßnahmen wie Ernährung oder körperliche Aktivität hin zu psychosozialen Fragestellungen $[3,6,13]$ gerecht zu werden. Diese soll- 
ten eine hohe Expertise und Glaubwürdigkeit aufweisen. Sowohl Printmedien als auch audiovisuelle Formate und Internetangebote kommen als ergänzende Informationsmedien infrage. Onlineangebote ermöglichen unter Einsatz moderner Techniken eine Individualisierung über Tailoring (Anpassung des Angebotes für verschiedene Nutzergruppen) und/oder Interaktivität. Bei der Erstellung solcher Angebote könnte dem von der Tumorart, dem jeweiligen Tumorstadium und der Therapiesituation abhängig wechselnden Bedarf $[4,11]$ ebenso Rechnung getragen werden wie dem anders gerichteten Informationsbedarf älterer Patienten [8]. Entsprechend gestaltete Informationsangebote könnten auch von Ärzten genutzt werden, um sich selber ergänzend zu informieren und diese Informationen an Patienten weiter zu geben. Diese Vorgehensweise findet bei Patienten nicht nur Akzeptanz, sondern erhöht auch deren Compliance [7].

Die Grenze eines internetbasierten Ansatzes liegt in der niedrigen Akzeptanz des Internets in der Altersgruppe über 60 Jahren, die den größten Anteil der Patienten mit Tumorerkrankungen stellt; eine Einschränkung die übereinstimmend in der Literatur beschrieben wird [7]. Ob sich dies als Folge der verbesserten Akzeptanz und häufigeren Nutzung von online Angeboten ändern wird, bleibt abzuwarten. Erst in einigen Jahren erreichen die Jahrgänge, die mit der beruflichen oder privaten Nutzung des Internets als regelmäßigem
Informationsmedium bereits vertraut sind das Alter ansteigender Inzidenz von Malignomen.

Eine besondere Herausforderung der Informationssuche im Internet ist die Vielfalt an Informationen und ihre für den Nutzer schwierig zu beurteilende Qualität [1]. Institutionen wie die Deutsche Krebshilfe und die Deutsche Krebsgesellschaft könnten hier eine wesentliche Aufgabe in der Gestaltung eines entsprechenden Angebotes haben, da Patienten bei Fachorganisationen Expertise und hohe Vertrauenswürdigkeit sehen [10]. Dabei sollte auf ein Angebot auch für Nutzer mit niedrigeren Bildungsabschlüssen geachtet werden, da sonst die Gefahr besteht, dass in dieser Gruppe die Nutzerrate deutlich abfällt [7].

\section{Konsequenzen für Klinik und Praxis}

- Das direkte Gespräch mit dem Arzt und der Pflegekraft ist die wesentliche Informationsquelle für Patienten mit Krebserkrankungen und ihre Angehörigen unabhängig von Alter.

- Diesem sollte im Gesundheitswesen durch Schaffung entsprechender zeitlicher wie räumlicher Gegebenheiten Rechnung getragen werden.

- Ärzte, Pflegekräfte und Institutionen sollten sich der Bedeutung des Gesprächs bewusst sein, die Rahmenbedingungen dafür schaffen und neben der fachlichen Expertise auch kommunikative Kompetenz erwerben bzw. fördern.

\section{Literatur}

1 Broom A. Virtually He@Ithy: The Impact of Internet Use on Disease Experience and the Doctor-Patient Relationship. Qual Health Res 2005; 15: 325-345

2 de Azambuja E, Ameye L, Paesmans M et al. The landscape of medical oncology in Europe by 2020. Ann Oncol 2014; 25: 525-528

3 Feldman-Stewart D, Brennenstuhl S, Brundage MD, Siemens DR. Overall information needs of early-stage prostate cancer patients over a decade: highly variable and remarkably stable; Support Care Cancer 2009; 17: 429-435

4 Gansler T, Kepner J, Willacy E et al. Evolving Information Priorities of Hematologic Cancer Survivors, Caregivers, and Other Relatives. J Canc Educ 2010; 25: 302-311

5 Gatta G, Mallone S, van der Zwan JM et al. Cancer prevalence estimates in Europe at the beginning of 2000. Ann Oncol 2013; 24: 1660-1666

6 Heimer A, Henkel M. Bedarf an Krebsinformation in der Bevölkerung: Analyse des Informationsverhaltens von Ratsuchenden. Repräsentative Befragung im Auftrag des Krebsinformationsdienstes des Deutschen Krebsforschungszentrums. www.krebsinformations dienst.de/info/krebsinformationsbedarf-2012bevoelkerung.pdf (Letzter Zugriff: 9.2.2015)

7 James N, Danielsy H, Rahmany R et al. A Study of Information Seeking by Cancer Patients and their Carers. Clinical Oncology 2007; 19: 356e-362e

8 Jansen J, van Weert J, van Dulmen S, Heeren T, Besning J. Patient education about treatment on cancer care. Cancer Nursing 2007; 30: 251-260

9 Lelorain S, Brédart A, Dolbeault S, Sultan S. A systematic review of the associations between empathy measures and patient outcomes in cancer care. Psychooncology 2012; 21: 1255-1264
10 Maddock C, Lewis I, Ahmad K, Sullivan R. Online information needs of cancer patients and their organizations. Ecancermedicalscience 2011; 5: 235

11 Mistry A, Wilson S, Priestman T et al. How do the information needs of cancer patients differ at different stages of the cancer journey? A cross-sectional survey. I R Soc Med Sh Rep 2010; 1: 30

12 Muusses LD, van Weert JC et al. Chemotherapy and information-seeking behaviour: characteristics of patients using mass-media information sources. Psychooncology 2012; 21: 993-1002

13 Nagler RH, Gray SW, Romantan A et al. Differences in information seeking among breast, prostate, and colorectal cancer patients: results from a population-based survey. Patient Educ Couns 2010; 81 Suppl: S54-S62

14 Bundesministerium für Gesundheit. Nationaler Krebsplan - Ziel 12a/12b/13 - Stärkung der kommunikativen Kompetenz der Leistungserbringer und der Patientenkompetenz. www.bmg.bund.de/ praevention/nationaler-krebsplan/was-haben-wirbisher-erreicht/ziel-12a12b13-staerkung-derkommunikativen-kompetenz-der-leistungserbringerund-der-patientenkompetenz.html (Letzter Zugriff: 9.2.2015)

15 Papadakos J, Bussière-Côté $\mathrm{S}$, Abdelmutti $\mathrm{N}$ et al. Informational needs of gynecologic cancer survivors. Gynecol Oncol 2012;124: 452-457

16 Robert Koch-Institut. Krebs in Deutschland. www.rki.de/DE/Content/Service/Presse/ Pressemitteilungen/2012/01_2012.html (Letzter Zugriff: 9.2.2015)
Interessenkonflikt

Die Autoren geben an, dass kein Interessenkonflikt besteht

DOI 10.1055/s-0041-100585 Dtsch Med Wochenschr 2015; 140: e43-e47 (c) Georg Thieme Verlag KG . Stuttgart · New York . ISSN 0012-0472 